\title{
Identification and pathogenicity of Alternaria species associated with leaf blotch disease and premature defoliation in French apple orchards
}

\author{
Kévin Fontaine ${ }^{1}$, Céline Fourrier-Jeandel ${ }^{1}$, Andrew D Armitage ${ }^{2}$, Anne-Laure Boutigny ${ }^{3}$, Manuela Crépet ${ }^{4}$, Valérie \\ Caffier $^{5}$, Dossi Carine Gnide ${ }^{1}$, Jason Shiller ${ }^{5}$, Bruno Le Cam ${ }^{5}$, Michel Giraud ${ }^{6}$, Renaud Ioos ${ }^{1}$, Jaime Aguayo ${ }^{\text {Corresp. } 1}$ \\ ${ }^{1}$ ANSES, Laboratoire de la Santé des Végétaux, Unité de Mycologie, USC INRAE 1480, Malzéville, France \\ 2 Natural Resources Institute, University of Greenwich, Chatham Maritime, Kent, United Kingdom \\ 3 ANSES, Laboratoire de la Santé des Végétaux, Unité Bactériologie, Virologie et OGM, Angers, France \\ 4 FREDON Rhône-Alpes, Saint-Priest, France \\ 5 Université d'Angers, Institut Agro, INRAE, IRHS, SFR QUASAV, F-49000 Angers, France \\ ${ }^{6}$ Centre opérationnel de Lanxade, CTIFL, Prigonrieux, France \\ Corresponding Author: Jaime Aguayo \\ Email address: jaime.aguayo@anses.fr
}

Leaf blotch caused by Alternaria spp. is a common disease in apple-producing regions. The disease is usually associated with one phylogenetic species and one species complex, Alternaria alternata and Alternaria arborescens species complex ( $A$. arborescens $\mathrm{SC}$ ), respectively. Both taxa may include the Alternaria apple pathotype, a quarantine or regulated pathogen in several countries. The apple pathotype is characterized by the production of a host-selective toxin (HST) which is involved in pathogenicity towards the apple. A cluster of genes located on conditionally dispensable chromosomes (CDCs) is involved in the production of this HST (namely AMT in the case of the apple pathotype). Since 2016, leaf blotch and premature tree defoliation attributed to Alternaria spp. have been observed in apple-producing regions of central and south-eastern France. Our study aimed to identify the Alternaria species involved in apple tree defoliation and assess the presence of the apple pathotype in French orchards. From 2016-2018, 166 isolates were collected and identified by multi-locus sequence typing (MLST). This analysis revealed that all these French isolates belonged to either the $A$. arborescens SC or $A$. alternata. Specific PCR detection targeting three genes located on the CDC did not indicate the presence of the apple pathotype in France. Pathogenicity was assessed under laboratory conditions on detached leaves of Golden Delicious and Gala apple cultivars for a representative subset of 28 Alternaria isolates. All the tested isolates were pathogenic on detached leaves of cultivars Golden Delicious and Gala, but no differences were observed between the pathogenicity levels of $A$. arborescens SC and $A$. alternata. However, the results of our pathogenicity test suggest that cultivar Golden Delicious is more susceptible than Gala to 
Alternaria leaf blotch. Implications in the detection of the Alternaria apple pathotype and the taxonomic assignment of Alternaria isolates involved in Alternaria leaf blotch are discussed. 
1 Identification and pathogenicity of Alternaria species

2

3

4

5 Kévin Fontaine ${ }^{1}$, Céline Fourrier-Jeandel ${ }^{1}$, Andrew D. Armitage ${ }^{2}$, Anne-Laure Boutigny ${ }^{3}$,

6 Manuela Crepet ${ }^{4}$, Valérie Caffier ${ }^{5}$, Dossi C. Gnide ${ }^{1}$, Jason Shiller ${ }^{5}$, Bruno Le Cam ${ }^{5}$, Michel

7 Giraud $^{6}$, Renaud $\operatorname{Ioos}^{1}$ and Jaime Aguayo ${ }^{1}$

8

9

10

11

12

13

\section{defoliation in French apple orchards}

${ }^{1}$ ANSES, Laboratoire de la Santé des Végétaux, Unité de Mycologie, USC INRAE 1480, Malzéville, France

${ }^{2}$ Natural Resources Institute, University of Greenwich, Chatham Maritime, Kent, United Kingdom

${ }^{3}$ ANSES, Laboratoire de la Santé des Végétaux, Unité Bactériologie, Virologie et OGM, Angers, France

${ }^{4}$ FREDON Rhône-Alpes, Saint-Priest, France

${ }^{5}$ IRHS-UMR1345, Université d'Angers, INRAE, Institut Agro, Beaucouzé, France

${ }^{6}$ Centre opérationnel de Lanxade, CTIFL, Prigonrieux, France

Corresponding Author:

Jaime Aguayo ${ }^{1}$

Domaine de Pixérécourt, Bât.E, 54220 Malzéville, France

jaime.aguayo@anses.fr 


\section{Abstract}

28

29

30

31

32

33

34

Leaf blotch caused by Alternaria spp. is a common disease in apple-producing regions. The disease is usually associated with one phylogenetic species and one species complex, Alternaria alternata and Alternaria arborescens species complex (A. arborescens SC), respectively. Both taxa may include the Alternaria apple pathotype, a quarantine or regulated pathogen in several countries. The apple pathotype is characterized by the production of a host-selective toxin (HST) which is involved in pathogenicity towards the apple. A cluster of genes located on conditionally dispensable chromosomes (CDCs) is involved in the production of this HST (namely AMT in the case of the apple pathotype). Since 2016, leaf blotch and premature tree defoliation attributed to Alternaria spp. have been observed in apple-producing regions of central and south-eastern France. Our study aimed to identify the Alternaria species involved in apple tree defoliation and assess the presence of the apple pathotype in French orchards. From 2016-2018, 166 isolates were collected and identified by multi-locus sequence typing (MLST). This analysis revealed that all these French isolates belonged to either the A. arborescens SC or A. alternata. Specific PCR detection targeting three genes located on the CDC did not indicate the presence of the apple pathotype in France. Pathogenicity was assessed under laboratory conditions on detached leaves of Golden Delicious and Gala apple cultivars for a representative subset of 28 Alternaria isolates. All the tested isolates were pathogenic on detached leaves of cultivars Golden Delicious and Gala, but no differences were observed between the pathogenicity levels of $A$. arborescens SC and A. alternata. However, the results of our pathogenicity test suggest that cultivar Golden Delicious is more susceptible than Gala to Alternaria leaf blotch. Implications in the detection of the Alternaria apple pathotype and the taxonomic assignment of Alternaria isolates involved in Alternaria leaf blotch are discussed. 


\section{Introduction}

53 Alternaria spp. are ubiquitous fungi comprising approximately 300 different species (Simmons

54 2007; Woudenberg et al. 2013). The genus has different lifestyles and can be isolated from a

55 large number of substrates (Thomma 2003; Woudenberg et al. 2013). Alternaria spp. are major

56 pathogenic fungi in agriculture and the food industry, leading to serious diseases in many

57 economically important crops (Meena et al. 2017; Thomma 2003).

58 Different taxa of Alternaria have been associated with Alternaria leaf blotch (ALB) and

59 Alternaria fruit spot (AFS) diseases (Armitage et al. 2015; Gur et al. 2017; Harteveld et al.

60 2013b). ALB is characterized by the development of round or irregular brown spots on leaves,

61 bordered by dark brown to purple margins (Rotondo et al. 2012). These symptoms generally start

62 in late spring or early summer, developing to yellowing leaves that can lead to early tree

63 defoliation and a premature fruit drop associated with a reduction in tree vigour and fruit quality

64 over the following years (Harteveld et al. 2013b; Rotondo et al. 2012). ALB may cause up to

$6580 \%$ of defoliation in some susceptible apple cultivars (Filajdić \& Sutton 1991) and

66 consequently may drastically decrease fruit yields (Harteveld et al. 2013b; Horlock 2006). Less

67 frequent, AFS is characterized by necrotic spots on the skin of the fruit surrounded by a red halo

68 centered on the lenticels (Harteveld et al. 2013b; Horlock 2006; Rotondo et al. 2012) and in

69 some cases can result in calyx cracking and fruit rot (Gur et al. 2017). AFS may consequently

70 downgrade the fruit's value, resulting in a significant financial burden to apple growers (Gur et

71 al. 2017; Harteveld et al. 2014).

72 Both ALB and AFS have been reported in nearly all apple-producing regions of the world

73 (Dickens \& Cook 1995; Filajdić \& Sutton 1991; Gur et al. 2017; Harteveld et al. 2013b; Kim et

74 al. 1986; Ozgonen \& Karaca 2006; Rotondo et al. 2012; Wenneker et al. 2018). Taxa causing 
75 ALB and AFS are part of the Alternaria section Alternaria that comprises the small-spored

76 Alternaria species. As for the whole genus Alternaria, identification of isolates within the

77 section Alternaria is challenging due to morphological plasticity and genetic similarity

78 (Armitage et al. 2015; Lawrence et al. 2013; Woudenberg et al. 2013). However recent advances,

79 especially in multi-gene phylogeny and comparative genomics, have allowed the different

80 Alternaria sections to be redefined and delineated, with accurate molecular differentiation and

81 identification of isolates (Armitage et al. 2020; Woudenberg et al. 2015a). Woudenberg et al.

82 (2015a), for example, have shown that the Alternaria section Alternaria consists of 11

83 phylogenetic species and one species complex. The taxonomic implications of this study are

84 major because 35 morphospecies, which could not be distinguished through multi-gene

85 phylogeny, were synonymized under Alternaria alternata (including the important plant

86 pathogens A. alternata, A. tenuissima and A. citri).

87 ALB and AFS have been commonly associated with the phylogenetic species $A$. alternata and 88 the Alternaria arborescens species complex (A. arborescens SC) (Gur et al. 2017; Harteveld et 89 al. 2013b; Rotondo et al. 2012; Toome-Heller et al. 2018; Wenneker et al. 2018), with both taxa 90 also known as saprophytic and generalist opportunistic pathogens affecting a variety of important 91 crops (Armitage et al. 2015; Thomma 2003). Both A. alternata and A. arborescens SC may 92 include the apple pathotype, which has been recently shown to be polyphyletic (Armitage et al. 93 2020). The apple pathotype, formerly known as Alternaria mali, causes significant problems in 94 apple orchards in south-eastern Asia (Li et al. 2019), was responsible for ALB in the south95 eastern USA in the early nineties (Filajdić \& Sutton 1991) and has been associated with severe 96 AFS in Israel (Gur et al. 2017). It is also listed either as a quarantine or a regulated pathogen in 97 several countries throughout the world (https://gd.eppo.int/). 
98 In Alternaria, pathotypes are characterized by the production of polyketide host-selective toxins

99 (HSTs), which are linked to pathogenicity affecting specific hosts (Tsuge et al. 2013). To date, at

100 least seven pathotypes have been described, each producing a unique HST essential to

101 pathogenicity in apples $(A M T)$, Japanese pears $(A K T)$, strawberries $(A F T)$, tangerines $(A C T)$,

102 tomatoes $(A A L)$ rough lemons $(A C R)$ and tobacco $(A T)$ (Tsuge et al. 2013; Wang et al. 2019).

103 The production of these HSTs involves a cluster of genes located on conditionally (or accessory)

104 dispensable chromosomes (CDCs), so named because they are not essential for saprophytic

105 growth and reproduction of pathogens (Hatta et al. 2002; Wang et al. 2019).

106 In the case of the apple pathotype, at least 17 genes could be involved in the synthesis of $A M T$

107 apple toxin (Harimoto et al. 2007) but so far only four, i.e. AMT1, AMT2, AMT3 and AMT4, have

108 been demonstrated to be involved in this process. To date, molecular detection of this pathotype

109 is only possible by PCR targeting one of the genes involved in the production of the $A M T$ apple

110 toxin (Armitage et al. 2020; Harimoto et al. 2007; Johnson et al. 2000) or by identifying these

111 genes in the genome of Alternaria isolates using bioinformatics (Armitage et al. 2020). However,

112 molecular taxonomic assignment to either A. alternata or the A. arborescens $\mathrm{SC}$ in routine

113 diagnostics requires the construction of multi-gene phylogenies (Armitage et al. 2015; Harteveld

114 et al. 2013b; Rotondo et al. 2012; Woudenberg et al. 2015a). Indeed, multi-locus sequence

115 typing (MLST) with relevant phylogenetic markers for the Alternaria genus has been used to

116 identify $A$. alternata and the $A$. arborescens SC. It has also enabled researchers to understand

117 their association with ALB and AFS in several countries (Armitage et al. 2015; Gur et al. 2017;

118 Harteveld et al. 2013b; Rotondo et al. 2012; Toome-Heller et al. 2018; Wenneker et al. 2018).

119 ALB has been observed for years in French orchards without causing serious damage. However,

120 since 2016, significant defoliation in trees infected by Alternaria has been reported in regions of 
121 central and south-eastern France. The reported presence of the apple pathotype in northern Italy

122 (Rotondo et al. 2012) has raised serious concerns for both the French plant health authorities and 123 apple growers.

124 The first objective of this study was to assess whether the upsurge in ALB symptoms observed in 125 French orchards was due to the emergence of the apple pathotype. To identify the pathogens

126 responsible for these unusual cases of defoliation, we conducted MLST analyses to determine the 127 phylogenetic position of the French Alternaria isolates. We also assessed the presence of the 128 apple pathotype by PCR tests targeting two genes involved in the production of the $A M T$ apple 129 toxin, and a gene found in the apple pathotype CDC that has homologs in the pear and 130 strawberry pathotypes (Armitage et al. 2020). Pathogenicity tests were then carried out and 131 Koch's postulates were assessed on the Gala and Golden Delicious apple cultivars using a 132 representative panel of isolates. Finally, both the phylogenetic position and pathogenicity of the 133 French Alternaria isolates were compared with Alternaria isolates from different countries 134 and/or isolated from crops other than apple.

\section{Materials \& Methods}

136 Isolate collection

137

French Alternaria spp. isolates (166) were obtained from symptomatic field samples of leaves 139 (156) and fruit (10) collected from ten different apple cultivars or cultivar groups (Table 1, 140 Supplementary material S1).Apple orchards were located in four major apple-producing regions 141 in central and south-eastern France: Auvergne-Rhône-Alpes (108 isolates), Provence-Alpes-

142 Côtes d'Azur (49 isolates), Occitanie (8 isolates) and Nouvelle-Aquitaine (1 isolate). The 143 samples were collected over 3 years (seasons 2016-17, 2017-18 and 2018-19, Table 1, 144 Supplementary material S1). Leaves and fruit surfaces were first disinfected with $70 \%$ ethanol 
145 and necrotic spots were excised using a sterile scalpel blade then plated onto Petri dishes

146 containing malt extract agar (Sigma-Aldrich) medium supplemented with chloramphenicol (0.2

$147 \mathrm{~g} / \mathrm{L})$. The cultures were incubated for four to seven days at $22{ }^{\circ} \mathrm{C}$ with a $12 \mathrm{~h}$ alternating dark and

148 light cycling period. A plug of each actively growing culture was then transferred to a new malt

149 extract agar Petri dish and incubated under the same conditions as described above. The isolate

150 collection was supplemented with 43 Alternaria isolates either associated with ALB or AFS

151 from Australia (16), Israel (8), Italy (14) and New Zealand (5). Furthermore, the Food and

152 Environmental Research Agency (FERA) contributed 12 isolates obtained from fruit

153 importations showing AFS and intercepted in the UK (unknown origin). Nine additional ALB or

154 AFS isolates were obtained from the Westerdijk Institute's collection (https://www.wi.knaw.nl).

155 Other Alternaria isolates associated with post-harvest apple rot problems in Argentina (4) and

156 South Africa (8) were also included. Additionally, the collection was completed with Alternaria

157 isolates from other hosts belonging to different botanical families (19 isolates). Five isolates in

158 the collection were identified as apple pathotype isolates. All the isolates were single-spored

159 before analysis. Details of the isolate collection studied in this work are presented in

160 Supplementary material S1.

161

162 Genomic DNA extraction and loci sequencing

163 DNA extractions were performed with approximately $0.5 \mathrm{~g}$ of Alternaria mycelium, scraped

164 from a fresh culture on malt extract, using the NucleoSpin Plant II kit (Macherey Nagel).

165 Mycelium was ground by placing two sterilized steel beads ( $3 \mathrm{~mm}$ in diameter) in an Eppendorf

166 tube containing mycelium with $400 \mu \mathrm{L}$ of lysis buffer and $10 \mu \mathrm{L}$ of RNAse (both provided with

167 the DNA extraction kit). Samples were subsequently ground twice for $60 \mathrm{~s}$ at $30 \mathrm{~Hz}$ in a MM 
168400 mixer mill (Retch). DNA was extracted according to the manufacturer's instructions. The 169 concentration of the DNA extracts (100 $\mu \mathrm{L}$ final volume) was estimated with a NanoDrop TM 1702000 Spectrophotometer (ThermoFisher). All the extracted DNA was stored at $-30^{\circ} \mathrm{C}$ until use.

171 The endopolygalacturonase (EndoPG) and the Alternaria major allergen (Alta-1) genes, two loci 172 commonly used in Alternaria identification and phylogenetics (Table 2, Armitage et al. 2015;

173 Harteveld et al. 2013b; Lawrence et al. 2013), were sequenced for all the isolates. In addition, the 174 anonymous region OPA 10-2 (Table 2, Rotondo et al. 2011, Woudenberg et al. 2015) was 175 sequenced for a subset of 100 isolates and used to assess putative differences in taxonomic 176 identification by comparison with EndoPG and Alta-1. For PCR amplification of the three loci, 177 the reaction mixtures contained 1X PCR reaction buffer (HGS Diamond Taq, Eurogentec), 2.5 $178 \mathrm{mM} \mathrm{MgCl}_{2}$ (4.0 mM for EndoPG), 4 x $0.25 \mathrm{mM}$ dNTPs, $0.2 \mu \mathrm{M}$ of forward and reverse primers 179 (Table 2), 1 U of HGS diamond Taq (Eurogentec), $2 \mu \mathrm{L}$ of DNA extract and molecular grade 180 water to complete up to $25 \mu \mathrm{L}$. PCR conditions consisted of an initial denaturation step at $95^{\circ} \mathrm{C}$ 181 for $10 \mathrm{~min}$, followed by 40 cycles at $94^{\circ} \mathrm{C}$ for $45 \mathrm{~s}$, annealing temperatures of $57^{\circ} \mathrm{C}$ for Alta- 1 , $18256^{\circ} \mathrm{C}$ for EndoPG and $62^{\circ} \mathrm{C}$ for OPA $10-2$ for $30 \mathrm{~s}$ (Table 2 ), $72^{\circ} \mathrm{C}$ for $1 \mathrm{~min}$ and a final 183 extension step at $72^{\circ} \mathrm{C}$ for $7 \mathrm{~min}$. The GENEWIZ sequencing platform (Leipzig, Germany) was 184 used for bidirectional Sanger sequencing of the amplicons. Consensus sequences were obtained 185 after manual correction using the Geneious R11 programme.

\section{Phylogenetic analysis}

188 The EndoPG, Alta-1 and OPA 10-2 sequence datasets generated in our study were supplemented 189 with data from previous studies. The sequence datasets that enabled taxonomic identification of 190 isolates in these previous studies (Armitage et al. 2015; Gur et al. 2017; Harteveld et al. 2013b;

191 Rotondo et al. 2012; Woudenberg et al. 2015a) were used as a reference in our phylogenetic 
192 analysis. As we performed molecular identification using an MLST approach, we decided to use

193 the taxonomy of the Alternaria section Alternaria proposed by Woudenberg et al. (2015a),

194 which consists of 11 phylogenetic species and one species complex. In other words, we used the

195 Alternaria alternata phylogenetic species without including any results of morphospecies (e.g. A.

196 tenuissima was taxonomically assigned to the phylogenetic species $A$. alternata), an approach

197 used in other studies that described isolates morphologically (Armitage et al. 2015; Rotondo et

198 al. 2012). DNA sequences were first analyzed with SeaView version 4 (Gouy et al. 2010). These

199 analyses included sequence alignments using MUSCLE (Edgar 2004) and elimination of poorly

200 aligned positions with Gblocks (Talavera \& Castresana 2007). MrBayes version 3.2 (Ronquist et

201 al. 2012b) was used for multi-locus phylogeny analysis on concatenated sequences for EndoPG

202 and Alta-1 (two-locus MSLT phylogenetic tree) and EndoPG, Alta-1 and OPA 10-2 (three-locus

203 MLST phylogenetic tree) separately. Runs were performed under the Bayesian MCMC model

204 jumping approach, which provides a convenient alternative to model selection before analysis

205 (command lset applyto= (all) nst=mixed). In model jumping, the Markov Chain Monte Carlo

206 (MCMC) sampler explores di $\square$ erent models and weights the results according to the posterior

207 probability of each model (Ronquist et al. 2012a). Four MCMC chains were run using the default

208 heating with tree sampling performed every 5000 generations. Runs were performed for at least

20920 million generations, and stopped when the standard deviation of split frequencies was below

2100.01 (Ronquist et al. 2012a). Homologous sequences of Alta-1 and Endo-PG for A. brassicicola

211 (isolate Abra43) were used as an outgroup in all the generated trees. The consensus tree was

212 obtained by using the command sumt. The resulting phylogenetic trees were visualised and

213 annotated with the interactive tree of life (iTOL) online tool (Letunic \& Bork 2016). The

214 taxonomic identification of 100 isolates using the concatenated trees EndoPg/Alta-1 and 
215 EndoPg/Alta-1/OPA 10-2 was compared with the function tanglegram implemented in

216 DENDROSCOPE 3.2.10 (Huson \& Scornavacca 2012). A subset of single-locus sequence data

217 for the corresponding loci was submitted to Genbank (accession nos. MN975269-MN975340,

218 Supplementary material S1).

219 PCR detection of the Alternaria apple pathotype

220

221 We searched for the Alternaria apple pathotype among all the French isolates by PCR targeting

222 two genes involved in AMT apple toxin biosynthesis — namely AMT1 and AMT2 — using

223 primers developed by Johnson et al. (2000) and Harimoto et al. (2007) respectively (Table 2).

224 Additionally, 44 out of these French isolates were also tested by PCR targeting AMT14, a gene

225 found in the apple pathotype toxin gene cluster and for which homologous genes also exist in

226 pear and strawberry pathotypes (Armitage et al. 2020). Other non-French isolates were tested by

227 PCR targeting either AMT1 / AMT2 or AMT1 / AMT2 / AMT14 genes (27 and 47 isolates

228 respectively, Supplementary material S1). PCRs were performed in $25-\mu \mathrm{L}$ reaction mixtures

229 containing 1X PCR reaction buffer (HGS Diamond Taq, Eurogentec), $2.5 \mathrm{mM} \mathrm{MgCl} 2,4 \times 0.25$

$230 \mathrm{mM}$ dNTPs, $0.2 \mu \mathrm{M}$ of forward and reverse primers (Table 2), $1 \mathrm{U}$ of HGS diamond Taq

231 (Eurogentec), $2 \mu \mathrm{L}$ of DNA extract and molecular grade water to complete up to $25 \mu \mathrm{L}$. PCR

232 conditions comprised an initial $10 \mathrm{~min}$ denaturation step at $95^{\circ} \mathrm{C}$ followed by 40 cycles of a

233 denaturation step at $94^{\circ} \mathrm{C}$ for $30 \mathrm{~s}$, an annealing step at $65^{\circ} \mathrm{C}$ for $A M T 1,57^{\circ} \mathrm{C}$ for $A M T 2,66^{\circ} \mathrm{C}$

234 for $A M T 14$ (Table 2) and an extension step at $72^{\circ} \mathrm{C}$ for $60 \mathrm{~s}$. These cycles were followed by a

235 final extension at $72^{\circ} \mathrm{C}$ for $7 \mathrm{~min}$. All PCRs were performed in duplicate. Controls were included

236 in all reactions. Positive controls included either gDNA (Alternaria apple pathotype isolate

237 LSVM 75) for AMT14 testing or a plasmid solution of $A M T 1$ and $A M T 2$ genes inserted in a 
238 vector using the pCR4-TOPO cloning kit (Invitrogen) following the manufacturer's instructions.

239 Negative controls consisted of sterile distilled water (SDW).

\section{Pathogenicity assays and Koch's postulates}

242 Twenty-eight isolates were used for pathogenicity assays: ten A. alternata, 17 A. arborescens SC

243 and one $A$. brassicicola (Supplementary material S1). Their selection took into account isolation

244 from different apple cultivars (10) and origin (5 countries) (Supplementary material S1). It also

245 covered different clades or subclades of the MLST phylogenetic tree constructed with the

246 EndoPG and Alta-1 regions (Figure 1). No apple pathotype isolate could be tested, because no

247 strain in our collection could produce a sufficient amount of conidia in culture, despite several

248 attempts. The $A$. brassicicola isolate Abra43 was included as a non-pathogenic control. Assays

249 were performed on detached apple leaves from Golden Delicious clone X972 and Gala clone

$250 \mathrm{X} 4712$, the most industrially-relevant cultivars in France, representing $45 \%$ of the total apple

251 production in the country (AGRESTE 2021). For simplicity, both clones will be referred

252 hereafter to as Golden Delicious and Gala. Spore suspensions were obtained from isolates grown

253 at $22^{\circ} \mathrm{C}$ for 21 days on malt extract agar medium under specific light condition cycles (Carvalho

254 et al. 2008): an incubation period of 7 days under a $12 \mathrm{~h}$ alternating dark then light cycling

255 period followed by 2 days under an 8 h-UV/16 h dark conditions (UV light-induced by a black

256 fluorescent near UV lamp (Philips/15W, T8-BLB) and a final cycle of 12 days in full darkness.

257 For each isolate, the inoculum was obtained by flooding the culture with $2 \mathrm{~mL}$ of SDW before

258 dislodging spores by scraping the plate with an L-shaped spreader. After a filtration step with

259 sterile gauze, the spore suspensions were counted and adjusted to a concentration of $1 \times 10^{5}$

260 conidia/mL with a haemocytometer. Leaves from the third or fourth node were detached from

261 fresh branches of apple saplings grown in a glasshouse. Eight leaves cleaned with 70\% ethanol 
262 were placed in plastic boxes containing two white absorbent paper towels humidified with SDW

263 and conserved at ambient temperature overnight before leaf inoculations. An experimental

264 replicate consisted of one strain inoculated on five different leaves (placed in five different

265 plastic boxes) per cultivar. Unwounded abaxial leaf surfaces were inoculated at six points with

$26610 \mu \mathrm{L}$ of conidial suspension. Each plastic box contained a negative control that consisted of one

267 leaf inoculated with SDW. Inoculated leaves were incubated at $20^{\circ} \mathrm{C}$ for 10 days under an

268 alternating $12 \mathrm{~h}$ dark then light cycling period. Each isolate was tested twice in independent

269 experiments. The results from each experiment were analyzed separately at the three data

270 collection times: 4, 7 and 10 days post-inoculation (dpi). Two types of analysis were performed.

271 For data from 4 dpi, a zero-inflated Poisson general linear mixed model (GLMM) was used to

272 assess the number of lesions per leaf. The model included the following explanatory variables:

273 the taxon of the tested isolate (A. alternata or A. arborescens SC), the apple cultivar (Gala or

274 Golden Delicious), and an experiment repeat variable (each isolate was tested twice in

275 independent experiments). An isolate effect was taken into account as a random variable. For

276 data from 7 and 10 dpi, zero-inflated beta GLMMs were performed. On both 7 and 10 dpi the

277 response variable was the proportion of the diseased leaf area on detached leaves that

278 corresponds to the lesion size. The diseased leaf area proportion (necrosis) was assessed by

279 visual inspection and coded between 0 and 1 . The model included the same explanatory and

280 random effect variables used for the 4 dpi data model: taxon, cultivar, repeat and isolate (random

281 effect). All GLMM analyses (on 4, 7 and 10 dpi) took into account only isolates of $A$. alternata

282 and A. arborescens SC, as only one A. brassicicola isolate was used, which is not enough to be

283 included in the isolate random effect. All the models were run in the R environment (version

284 4.0.3) using the glmmTMB package (Brooks et al. 2017). Excess zeros were in all cases tested 
285 with the function testZeroInflation of the DHARMa R package (Hartig 2017), which compares

286 the distribution of expected zeros in the data with the observed zeros. Model residual diagnostics

287 of all models were performed with the DHARMa package. Analysis of variance type II

288 (ANOVA type II) was performed to assess the effect of each explanatory variable. Koch's

289 postulates were assessed by the re-isolation on malt agar extract medium of 23 randomly chosen

290 tested isolates (Supplementary material S1) and the re-sequencing of Alta-1 and EndoPG loci.

\section{Results}

\section{Molecular identification of strains}

293 Concatenation of Alt-a1 and Endo-PG sequences resulted in a 900-bp alignment. This alignment

294 was used for phylogenetic analyses. Depending on the isolate, the number of bases/residues that

295 differed between isolates of $A$. alternata and isolates of other taxa of the Alternaria section

296 Alternaria included in the analysis (A. arborescens SC, A. gaisen, A. longipes, A. gossypina and

297 A. alstroermeriae) ranged from 6 to 37. The two-marker phylogenetic tree distinguished two major

298 clades: A. alternata and A. arborescens SC (Figure 1). The A. alternata phylogenetic clade

299 encompassed four subclades, while the $A$. arborescens SC encompassed two. The analysis could

300 also distinguish these two clades from other taxa in the Alternaria section Alternaria: A. gaisen,

301 A. longipes, A. gossypina and A. alstroemeriae (Figure 1). The three concatenated genes (Alta-

302 1/Endo-PG/OPA10-2) resulted in a 1534-bp alignment which included 18-65 differences/residues

303 between A. alternata and other taxa of the Alternaria section Alternaria included in the analysis.

304 As for the two-marker concatenated tree, the phylogenetic analysis using three markers

305 distinguished two major clades, A. alternata and A. arborescens SC (Supplementary material S2).

306 It could also distinguish these clades from other taxa in the Alternaria section Alternaria (A.

307 gaisen, A. longipes, A. gossypina and A. alstroemeriae). The phylogenetic tree pattern was similar 
308 to that determined with two loci (i.e. Alta-1 and EndoPG). The A. alternata clade was divided into

309 four subclades, whereas the $A$. arborescens SC was separated into two subclades. Adding a third

310 locus to the analysis (OPA 10-2) did not improve the resolution within A. alternata and $A$.

311 arborescens SC (Supplementary material S2). The two phylogenetic analyses did, however, refine

312 identification: two Australian strains were consequently assigned to A. alternata whereas they had

313 previously been assigned to A. longipes (BRIP46356 and BRIP46455) by Harteveld et al. (2013b)

314 (Figure 1, Supplementary materiel S1).

315

316

317

318

319

320

321

322

323

324

325

326

327

328

329

330

331

\section{Identification of Alternaria isolates causing ALB in France}

Isolates from France were identified as either $A$. arborescens SC (91 isolates, 55\%) or $A$. alternata (75 isolates, $45 \%$ ) based on the taxonomic identification with the Alta-1 and EndoPG markers. No changes in the taxonomic identification were observed for the subset of isolates with sequences of Alta-1, EndoPG and OPA 10-2 markers (Supplementary material S1). The distribution of isolates differed according to the cultivar (Table 1). It was observed that $A$. arborescens SC isolates were more frequent on Gala and Golden Delicious cultivars, whereas A. alternata isolates were more frequent on cultivars Reinette grise du Canada, Dalinette and Crimson. On Braeburn and Pink lady cultivars, there was a similar number of isolates of $A$. arborescens $\mathrm{SC}$ and A. alternata. On Garance and GoldRush (Coop38cov) cultivars, too few isolates were recovered to make any comparison (Table 1).

\section{Screening for the Alternaria apple pathotype}

None of the 166 French isolates were identified as the apple pathotype by PCR tests targeting the $A M T$ apple toxin (AMT1, AMT2) or cross-pathotype (AMT14) loci (Supplementary material S1). All five apple pathotype reference isolates behaved as expected and yielded positive results for 
332 333

334 335 336 337 338 339 340

AMT1 and AMT2 PCR tests and also for the tests targeting AMT14, which is common to apple, pear and strawberry pathotypes (Supplementary material S1). However, four isolates formerly identified as apple pathotype in earlier studies in Italy (Rotondo et al. 2012) and Israel (Gur et al. 2017) gave negative PCR results for the three loci in our conditions, thus overturning their identification (Supplementary material S1).

\section{Pathogenicity assays and Koch's postulates}

At 4 dpi, 27 isolates - including the negative control $A$. brassicicola - were able to induce at least one necrotic spot on detached leaves of apple cultivars Golden Delicious and Gala. The only exception was an $A$. arborescens SC isolate (16_489b3a) that did not induce necrotic spots on detached Gala cultivar leaves. The zero-inflated Poisson GLMM used to assess the number of lesions per leaf on 4 dpi, indicated a significant effect of the experiment repetition (Type II Wald; $\left.\chi^{2}=17.75 ; \mathrm{df}=2 ; \mathrm{p}=0.00014\right)$ but not of the taxa (Type II Wald; $\chi^{2}=2.44 ; \mathrm{df}=1 ; \mathrm{p}=0.118$ ) or the apple cultivar (Type II Wald; $\chi^{2}=0.06 ; \mathrm{df}=1 ; \mathrm{p}=0.810$ ) (Figure 2). All 28 tested isolates were able to induce leaf blotch after 7 dpi on Golden Delicious and Gala. On 7 dpi, the zero-inflated beta GLMM used to assess the proportion of the diseased leaf area indicated a significant effect of the experiment repetition (Type II Wald; $\chi^{2}=84.013$; $\mathrm{df}=2 ; \mathrm{p}<0.001$ ) and the apple cultivar (Type II Wald; $\chi^{2}=17.296 ; \mathrm{df}=1 ; \mathrm{p}=3.199 \mathrm{e}^{-5}$ ), but not of the taxa (Type II Wald; $\chi^{2}=0.001 ; \mathrm{df}=1 ; \mathrm{p}=0.97$ ) (Figure 3a). On 10 dpi the zero-inflated beta GLMM indicated a significant effect of the experiment repetition (Type II Wald; $\chi^{2}=91.82 ; \mathrm{df}=2 ; \mathrm{p}<2.2 \mathrm{e}^{-16}$ ) and the apple cultivar (Type II Wald; $\chi^{2}=11.80 ; \mathrm{df}=1 ; \mathrm{p}=0.0006$ ), but not of the taxa (Type II Wald; $\chi^{2}=0.06 ; \mathrm{df}=1 ; \mathrm{p}=0.801$ ) (Figure 3b). On 7 and 10 dpi, leaves of the Golden Delicious cultivar were more susceptible than those of Gala, as measured by the proportion of the diseased leaf area (Figure 3). Raw 
355 356

361

362

363

364

365

366

367

368

369

370

371

372 373

374

375

376

377

measurements at 4, 7 and 10 dpi are presented in the supplementary material S4 section. Finally, the identity of 23 of these strains was confirmed by re-isolating and sequencing (Alta-1 and EndoPG loci), fulfilling Koch's postulates (Supplementary material S1). No disease symptoms were observed on leaves inoculated with water. Examples of the results from the pathogenicity tests are shown in the supplementary material S5 section.

\section{Discussion}

\section{The Alternaria apple pathotype was not found in French orchards}

We firstly checked whether we were witnessing the emergence of the apple pathotype in French orchards. We did this through PCR assays targeting three genes located in the conditionally dispensable chromosome (CDC) - AMT1 (Johnson et al. 2000), AMT2 (Harimoto et al. 2008) and AMT14 (Armitage et al. 2020) — which characterizes isolates of the apple pathotype. Our results showed that this pathogen was not present in France within the sampled regions and years. To date, molecular detection of the apple pathotype has only been possible by PCR tests that target genes present in the CDC. These targets are associated with secondary metabolite clusters involved in the production of the apple pathotype host-specific toxin AMT (Armitage et al. 2020; Harimoto et al. 2007; Johnson et al. 2000). Although all our apple pathotype reference strains gave positive results using the three markers, the Italian and Israeli strains previously identified as apple pathotype by PCR based on the amplification of a modified PCR test targeting AMT1 (Rotondo et al. 2012) and AMT3 (Gur et al. 2017) gave negative results in our study with tests targeting loci $A M T 1, A M T 2$ and $A M T 14$. In the case of the Israeli strains, the primers targeting AMT3 (Harimoto et al. 2007), showed unsatisfactory results in our preliminary tests as several unexpected bands appeared after gel electrophoresis of the PCR product (data not shown) 
378 and these primers were discarded for subsequent molecular tests. The results obtained with the

379 Italian strains are more difficult to explain because the initial study of Rotondo et al. (2012)

380 performed several confirmation tests (including sequencing of the products). One hypothesis that

381 may explain the difficulty in amplifying these loci is the occurrence of partial or total

382 chromosomal loss in isolates. This phenomenon has previously been reported in the apple

383 pathotype by Johnson et al. (2001) and is due to chromosomal instability in culture. To avoid this

384 problem, in the analysis of French isolates, our tests targeting AMT1, AMT2 and AMT14 were

385 performed right after isolation, avoiding several subculturing cycles. However, in all the cases

386 where the presence of $A M T 1, A M T 2$ and $A M T 14$ was assessed (in the five reference isolates;

387 Supplementary material S1), all three gene-specific PCR assays gave positive results. Based on

388 the results of our study, we suggest that the apple pathotype should be detected from pure

389 cultures by using at least two or more of the existing molecular tests to target $A M T 1$ and AMT2,

390 which is a good option if the objective is to specifically detect the apple pathotype. By

391 optimizing and validating current tools or developing new molecular tests, it might be possible to

392 detect diseases in planta from symptomatic leaves, which could avoid isolate subculturing cycles

393 while minimizing the risk of chromosomal loss.

394

395 Co-existence of Alternaria alternata and the Alternaria arborescens species complex in

396 French orchards

397 The second objective of this study was to identify Alternaria species or groups associated with

398 Alternaria leaf blotch (ALB) and Alternaria fruit spot (AFS) in French orchards. The

399 phylogenetic trees generated after using MLST clearly showed that these diseases are caused by

400 two phylogenetic clades: A. alternata and A. arborescens SC, regardless of the apple cultivar. 
401 Our results also showed that both taxa may co-exist in the same orchard. These results confirm

402 that these two Alternaria taxa are the major cause of ALB and AFS in regions of the world

403 where these diseases have been studied so far (Gur et al. 2017; Harteveld et al. 2013b; Rotondo

404 et al. 2012; Toome-Heller et al. 2018; Wenneker et al. 2018). In addition, our results suggest that

405 sequencing two loci, i.e. Alta-1 and EndoPG, is enough to be able to distinguish Alternaria

406 isolates involved in these diseases. Firstly, these two loci enable the two major phylogenetic

407 clades - A. alternata and A. arborescens SC — to be distinguished. Secondly, the loci also

408 clearly distinguish these two clades from other Alternaria taxa within the Alternaria section.

409 Including the OPA 10-2 locus did not substantially improve the molecular identification of the

410 strains.

411

412 Alternaria alternata and the Alternaria arborescens SC are responsible for defoliation in

413 French apple orchards

414 We showed that the isolates collected from necrotic leaves were able to produce symptoms on

415 detached apple leaves of cultivars Gala and Golden Delicious. The latter cultivar was more

416 susceptible under our conditions, as shown by measurements of the diseased leaf area after 7 and

$41710 \mathrm{dpi}$, a quantitative trait generally used to measure pathogen aggressiveness. Gala and Golden

418 Delicious were used for pathogenicity tests since they are the most important cultivars in France.

419 Although these cultivars are considered as relatively "susceptible" to ALB and AFS (Filajdić \&

420 Sutton 1991; Harteveld et al. 2014; Rotondo et al. 2012), earlier studies have shown that there is

421 little, or no cultivar specificity in Alternaria taxa causing ALB and AFS, at least for the most

422 economically important apple cultivars used worldwide (Filajdić \& Sutton 1991; Harteveld et al.

423 2014; Li et al. 2019). Management of the disease may involve resistant cultivars. However, 
424 further research involving more cultivars would be required to assess potential cultivar-

425 specificity among Alternaria taxa causing ALB and AFS. The selection of disease-resistant

426 cultivars should rely upon studies combining data collected from the field and from trials under

427 controlled conditions (Li et al. 2019).

428 Our results also showed that the entire subset of Alternaria isolated from apple leaves or fruit

429 fulfilled Koch's postulates. The pathogenicity tests showed that there are no significant

430 differences between isolates of $A$. alternata and A. arborescens SC as assessed by the number of

431 lesions per leaf on 4 dpi or the proportion of the diseased leaf area on 4 and 7 dpi. Both results

432 are in agreement with previous studies that suggest that pathogenicity may be isolate-dependent

433 rather than species-dependent (Harteveld et al. 2014; Rotondo et al. 2012). One of the limits of

434 our study is that we could not assess the pathogenicity of any of the reference apple pathotype

435 strains because too few spores could be obtained during cultivation. It is important to highlight,

436 however, that previous studies comparing the pathogenicity of apple pathotype isolates with

437 other Alternaria isolates in apples have shown discrepant results: while Armitage et al. (2020)

438 showed that apple pathotype strains were significantly more pathogenic than other isolates that

439 do not carry CDCs, Rotondo et al. (2012) did not observe any difference in levels of

440 pathogenicity between apple pathotype isolates and other Alternaria isolated from apple leaves

441 or fruit. Unexpectedly, we observed symptoms on apple leaves inoculated by A. brassicicola,

442 which has never been reported as pathogenic on apples to our knowledge. These results suggest

443 that Alternaria isolates from other Alternaria sections that do not carry CDCs involved in the

444 production of HTS may also cause ALB symptoms under controlled conditions. This is probably

445 associated with the production of nonspecific Alternaria toxins that can affect many plants

446 regardless of whether they are or are not a host of the pathogen (Tsuge et al. 2013). However, as 
447 shown here and elsewhere, under natural conditions only small-spore Alternaria (Alternaria

448 section Alternaria) have so far been described as apple pathogens causing ALB and AFS. Recent

449 genomic resources, including the genome of A. brassicicola (Belmas et al. 2018) and isolates of

450 Alternaria involved in AFS and ALB (Armitage et al. 2020) will allow comparative genomics

451 analysis that may clarify these pathogenicity mechanisms.

452 Finally, this study identified the Alternaria taxa involved in ALB and AFS in France, but did not 453 determine the cause of the increased severity in these diseases over recent years (e.g.

454 introduction of the apple pathotype). However, alternative explanations may be suggested based 455 on previous epidemiological studies. Firstly, it seems that the disease develops better in 456 relatively hot (between $>20^{\circ} \mathrm{C}$ and $30^{\circ} \mathrm{C}$ ) and rainy weather (Bhat et al. 2015; Filajdić \& Sutton 457 1992; Harteveld et al. 2013a; Kim et al. 1986). Potential changes in these two parameters, or 458 other climatic factors, should be studied in greater depth in the French regions concerned by 459 AFS. Another hypothesis is the introduction of more virulent strains. This could occur by the 460 long-distance movement of spores carried by wind currents that may have transported Alternaria 461 air inoculum into apple orchards from sources in other apple-producing regions (Fernández462 Rodríguez et al. 2015; Woudenberg et al. 2015b). Finally, the emergence of fungicide resistance 463 among strains should not be ruled out, considering that apple orchards are treated intensely with 464 fungicides, mainly used to control apple scab caused by Venturia inaequalis, which also 465 contributes to the control of ALB and AFS (Horlock 2006).

\section{Conclusions}

468 Since 2016, Alternaria leaf blotch and premature defoliation attributed to Alternaria spp. have 469 been observed in apple-producing regions in central and south-eastern France. The emergence of 470 the Alternaria apple pathotype was suspected following its observation in northern Italy. The 
471 presence of the apple pathotype in French orchards was therefore assessed by a specific PCR

472 targeting three genes located on conditionally dispensable chromosomes across a large collection

473 of Alternaria isolates. Our results showed that the Alternaria apple pathotype was not present.

474 Taxonomic identification of these isolates, assessed by multi-locus sequence typing and

475 construction of phylogenetic trees, indicates that Alternaria leaf blotch in France is associated

476 with isolates of A. alternaria and A. arborescens SC. Pathogenicity tests of a subsample of

477 isolates demonstrated that they were all able to induce necrotic symptoms on detached apple

478 leaves of the cultivars Gala and Golden Delicious. Our results also showed that there are no

479 significant differences in levels of pathogenicity between isolates of A. alternata and A.

480 arborescens $\mathrm{SC}$. Our controlled pathogenicity tests do suggest, however, that cultivar Golden

481 Delicious is more susceptible to Alternaria leaf blotch. In the future, genetic and epidemiological 482 approaches are required to clarify why Alternaria leaf blotch events have increased in frequency

483 and severity in some regions of France.

484 Acknowledgments

485

486 The authors acknowledge the financial support of the French Ministry of Agriculture and the

487 French Agency for Biodiversity, ECOPHYTO/AFB, AAP CASDAR (call for projects 2017)

488 recherche technologique 1716 CREATIVE. The mycology research unit of the ANSES Plant

489 Health Laboratory (LSV) is supported by a grant managed by the French National Research

490 Agency (ANR) as part of the French government's "Investing for the Future" (PIA) program

491 (ANR-11-LABX-0002-01, Laboratory of Excellence-ARBRE).

492 We would also like to thank the French apple producers who supplied leaf and fruit samples, and

493 are grateful to Pr. Thomas Guillemette, Pr. Barry Pryor, Pr. Andrea Patriarca, Dr. Olufemi A.

494 Akinsanmi, Dr. Moshe Reuveni, Dr. Lior Gur, Dr. Marina Collina and Ms. Jacqueline Hubert for 
495

496

497

498

499

500

501

502

503

504

505

506

507

508

509

510

511

512

513

514

515

516

517

518

519

520

521

522

523

524

525

526

527

528

529

530

531

532

533

534

535

536

537

538

sharing Alternaria strains. We are also grateful to the LSV ANSES team members who helped to

perform some of the experiments, in particular Maurane Pagniez for the purification of single-

spore strains and Eugenie Vuittenez for the pathogenicity assays.

\section{References}

AGRESTE. 2021. En 2021, une production française de pommes prévue inférieure à la moyenne 2016-2020. Available at https://agreste.agriculture.gouv.fr/agresteweb/download/publication/publie/IraFru21105/2021_105inforappomme.pdf (accessed Pomme - N²/5 2021-105).

Armitage AD, Barbara DJ, Harrison RJ, Lane CR, Sreenivasaprasad S, Woodhall JW, and Clarkson JP. 2015. Discrete lineages within Alternaria alternata species group: Identification using new highly variable loci and support from morphological characters. Fungal Biology 119:994-1006. http://dx.doi.org/10.1016/j.funbio.2015.06.012

Armitage AD, Cockerton HM, Sreenivasaprasad S, Woodhall J, Lane CR, Harrison RJ, and Clarkson JP. 2020. Genomics Evolutionary History and Diagnostics of the Alternaria alternata Species Group Including Apple and Asian Pear Pathotypes. Frontiers in Microbiology 10. 10.3389/fmicb.2019.03124

Belmas E, Briand M, Kwasiborski A, Colou J, N'Guyen G, lacomi B, Grappin P, Campion C, Simoneau P, Barret M, and Guillemette T. 2018. Genome sequence of the necrotrophic plant pathogen Alternaria brassicicola Abra43. Genome Announc 6.

Bhat K, Peerzada S, and Anwar A. 2015. Alternaria epidemic of apple in Kashmir. African Journal of Microbiology Research 9:831-837.

Brooks ME, Kristensen K, van Benthem KJ, Magnusson A, Berg CW, Nielsen A, Skaug HJ, Machler M, and Bolker BM. 2017. glmmTMB balances speed and flexibility among packages for zero-inflated generalized linear mixed modeling. The $R$ journal 9:378-400.

Carvalho DD, Alves E, Batista TR, Camargos RB, and Lopes EA. 2008. Comparison of methodologies for conidia production by Alternaria alternata from citrus. Brazilian Journal of Microbiology 39:792-798.

Dickens JSW, and Cook RTA. 1995. Japanese pear black spot and apple Alternaria blotch. EPPO Bulletin 25:651-659. 10.1111/j.1365-2338.1995.tb01117.x

Edgar RC. 2004. MUSCLE: multiple sequence alignment with high accuracy and high throughput. Nucleic Acids Research 32:1792-1797. 10.1093/nar/gkh340

Fernández-Rodríguez S, Sadyś M, Smith M, Tormo-Molina R, Skjøth CA, Maya-Manzano JM, Silva-Palacios I, and Gonzalo-Garijo Á. 2015. Potential sources of airborne Alternaria spp. spores in south-west Spain. Science of The Total Environment 533:165-176.

Filajdić N, and Sutton T. 1991. Identification and distribution of Alternaria mali on apples in North Carolina and susceptibility of different varieties of apples to Alternaria blotch. Plant Disease 75:1045-1048.

Filajdić N, and Sutton T. 1992. Influence of temperature and wetness duration on infection of apple leaves and virulence of different isolates of Alternaria mali. Phytopathology 82:1279-1283.

Gouy M, Guindon S, and Gascuel O. 2010. SeaView version 4: a multiplatform graphical user interface for sequence alignment and phylogenetic tree building. Mol Biol Evol 27:221224. 
539

540

541

542

543

544

545

546

547

548

549

550

551

552

553

554

555

556

557

558

559

560

561

562

563

564

565

566

567

568

569

570

571

572

573

574

575

576

577

578

579

580

581

582

583

584

585

586

587

588

Gur L, Reuveni M, and Cohen Y. 2017. Occurrence and etiology of Alternaria leaf blotch and fruit spot of apple caused by Alternaria alternata f. sp. mali on cv. Pink lady in Israel. European Journal of Plant Pathology 147:695-708. 10.1007/s10658-016-1037-0

Harimoto Y, Hatta R, Kodama M, Yamamoto M, Otani H, and Tsuge T. 2007. Expression Profiles of Genes Encoded by the Supernumerary Chromosome Controlling AM-Toxin Biosynthesis and Pathogenicity in the Apple Pathotype of Alternaria alternata. Molecular Plant-Microbe Interactions 20:1463-1476. 10.1094/MPMI-20-12-1463

Harimoto Y, Tanaka T, Kodama M, Yamamoto M, Otani H, and Tsuge T. 2008. Multiple copies of AMT2 are prerequisite for the apple pathotype of Alternaria alternata to produce enough AM-toxin for expressing pathogenicity. Journal of General Plant Pathology 74:222-229. 10.1007/s10327-008-0089-1

Harteveld D, Akinsanmi OA, Chandra K, and Drenth A. 2013a. Timing of Infection and Development of Alternaria Diseases in the Canopy of Apple Trees. Plant Disease 98:401-408. 10.1094/PDIS-06-13-0676-RE

Harteveld D, Akinsanmi OA, and Drenth A. 2013b. Multiple Alternaria species groups are associated with leaf blotch and fruit spot diseases of apple in Australia. Plant Pathology 62:289-297. 10.1111/j.1365-3059.2012.02637.x

Harteveld D, Akinsanmi OA, and Drenth A. 2014. Pathogenic variation of Alternaria species associated with leaf blotch and fruit spot of apple in Australia. European Journal of Plant Pathology 139:789-799. 10.1007/s10658-014-0433-6

Hartig F. 2017. DHARMa: residual diagnostics for hierarchical (multi-level/mixed) regression models. $R$ package version 015.

Hatta R, Ito K, Hosaki Y, Tanaka T, Tanaka A, Yamamoto M, Akimitsu K, and Tsuge T. 2002. A conditionally dispensable chromosome controls host-specific pathogenicity in the fungal plant pathogen Alternaria alternata. Genetics 161:59-70.

Horlock CM. 2006. Management of Alternaria leaf and fruit spot in apples: Horticulture Australia. Huson DH, and Scornavacca C. 2012. Dendroscope 3: an interactive tool for rooted phylogenetic trees and networks. Systematic biology 61:1061-1067.

Johnson L, Johnson RD, Akamatsu H, Salamiah A, Otani H, Kohmoto K, and Kodama M. 2001. Spontaneous loss of a conditionally dispensable chromosome from the Alternaria alternata apple pathotype leads to loss of toxin production and pathogenicity. Current Genetics 40:65-72.

Johnson R, Johnson L, Kohmoto K, Otani H, Lane CR, and Kodama M. 2000. A Polymerase Chain Reaction-Based Method to Specifically Detect Alternaria alternata Apple Pathotype (A. mali), the Causal Agent of Alternaria Blotch of Apple. Phytopathology 90:973-976. 10.1094/PHYTO.2000.90.9.973

Kim C-H, Cho W-D, and Kim S-C. 1986. An empirical model for forecasting Alternaria leaf spot in apple. Korean journal of applied entomology 25:221-228.

Lawrence DP, Gannibal PB, Peever TL, and Pryor BM. 2013. The sections of Alternaria: formalizing species-group concepts. Mycologia 105:530-546. 10.3852/12-249

Letunic I, and Bork P. 2016. Interactive tree of life (iTOL) v3: an online tool for the display and annotation of phylogenetic and other trees. Nucleic Acids Research 44:W242-W245.

Li Y, Hu X-L, Trigiano R, Aldwinckle H, and Cheng Z-M. 2019. Evaluation of 110 Apple Cultivars for Resistance to Alternaria Blotch Caused by Alternaria alternata Apple Pathotype. HortScience 54:1268-1274. 10.21273/HORTSCI13841-18

Meena M, Gupta SK, Swapnil P, Zehra A, Dubey MK, and Upadhyay RS. 2017. Alternaria Toxins: Potential Virulence Factors and Genes Related to Pathogenesis. Frontiers in Microbiology 8:1451.

Ozgonen H, and Karaca G. 2006. First report of Alternaria mali causing necrotic leaf spot of apples in Turkey. Plant Pathology 55:578-578.

Peer) reviewing PDF | (2021:07:63284:2:0:NEW 18 Oct 2021) 
589

590

591

592

593

594

595

596

597

598

599

600

601

602

603

604

605

606

607

608

609

610

611

612

613

614

615

616

617

618

619

620

621

622

623

624

625
Ronquist F, Huelsenbeck J, and Teslenko M. 2012a. MrBayes version 3.2 manual: tutorials and model summaries. Available with the software distribution at mrbayessourceforgenet/mb32_manualpdf.

Ronquist F, Teslenko M, Van Der Mark P, Ayres DL, Darling A, Höhna S, Larget B, Liu L, Suchard MA, and Huelsenbeck JP. 2012b. MrBayes 3.2: efficient Bayesian phylogenetic inference and model choice across a large model space. Systematic biology 61:539-542.

Rotondo F, Collina M, Brunelli A, and Pryor BM. 2012. Comparison of Alternaria spp. collected in Italy from apple with $A$. mali and other AM-toxin producing strains. Phytopathology 102:1130-1142. 10.1094/PHYTO-04-12-0076-R

Simmons EG. 2007. Alternaria: An indentification manual. Utrecht, The Netherlands: CBS Fungal Biodiversity Centre.

Talavera G, and Castresana J. 2007. Improvement of phylogenies after removing divergent and ambiguously aligned blocks from protein sequence alignments. Systematic biology 56:564-577.

Thomma BPHJ. 2003. Alternaria spp.: from general saprophyte to specific parasite. Molecular Plant Pathology 4:225-236. 10.1046/j.1364-3703.2003.00173.x

Toome-Heller M, Baskarathevan J, Burnip G, and Alexander B. 2018. First report of apple leaf blotch caused by Alternaria arborescens complex in New Zealand. New Zealand Journal of Crop and Horticultural Science:1-6.

Tsuge T, Harimoto Y, Akimitsu K, Ohtani K, Kodama M, Akagi Y, Egusa M, Yamamoto M, and Otani H. 2013. Host-selective toxins produced by the plant pathogenic fungus Alternaria alternata. FEMS Microbiology Reviews 37:44-66. 10.1111/j.1574-6976.2012.00350.x

Wang M, Fu H, Shen X-X, Ruan R, Rokas A, and Li H. 2019. Genomic features and evolution of the conditionally dispensable chromosome in the tangerine pathotype of Alternaria alternata. Molecular Plant Pathology 20:1425-1438. https://doi.org/10.1111/mpp.12848

Wenneker M, Pham K, Woudenberg J, and Thomma BP. 2018. First report of Alternaria arborescens species complex causing leaf blotch and associated premature leaf drop of 'Golden delicious' apple trees in the Netherlands. Plant Disease 102:1654.

Woudenberg J, Seidl M, Groenewald J, de Vries M, Stielow J, Thomma B, and Crous P. 2015a. Alternaria section Alternaria: Species, formae speciales or pathotypes? Studies in Mycology 82:1-21.

Woudenberg J, Van Der Merwe N, Jurjević Ž, Groenewald J, and Crous P. 2015b. Diversity and movement of indoor Alternaria alternata across the mainland USA. Fungal Genetics and Biology 81:62-72.

Woudenberg JHC, Groenewald JZ, Binder M, and Crous PW. 2013. Alternaria redefined. Studies in Mycology 75:171-212. http://dx.doi.org/10.3114/sim0015 


\section{Table $\mathbf{1}$ (on next page)}

Distribution of Alternaria isolates obtained from French orchards from years 2016-2019.

The table shows the apple cultivar, the number of samples, the taxa (Alternaria arborescens $\mathrm{SC}$ or Alternaria alternata) and the co-occurrence of the isolates in the same orchard. The samples were identified by sequencing EndoPg and Alta-1. a: cultivars Galastar and Royal Gala included, b: cultivar Reinette grise du Canada (Canada), c: cultivar Crimson Crisp included and d: cultivar Rosy Glow included. 
1 Table 1. Distribution of Alternaria isolates obtained from French orchards from years 2016-2019.

2 The table shows the apple cultivar, the number of samples, the taxa (Alternaria arborescens SC or 3 Alternaria alternata) and the co-occurrence of the isolates in the same orchard. The samples were 4 identified by sequencing EndoPg and Alta-1. a: cultivars Galastar and Royal Gala included, b: 5 cultivar Reinette grise du Canada (Canada), c: cultivar Crimson Crisp included and d: cultivar 6 Rosy Glow included.

\begin{tabular}{ccccc}
\hline Cultivar & $\begin{array}{c}\text { No. of samples (including } \\
\text { fruit samples) }\end{array}$ & $\begin{array}{c}\text { A. } \\
\text { arborescens } \\
\text { species } \\
\text { complex }\end{array}$ & A. alternata & $\begin{array}{c}\text { Co-occurrence/sample } \\
\text { (including fruit samples) }\end{array}$ \\
\hline $\begin{array}{c}\text { Braeburn } \\
\text { Gala }\end{array}$ & 2 & 4 & 4 & 1 \\
Golden $_{\text {Delicious }}$ & $13(2)$ & 16 & 8 & 3 \\
Canada & 10 & 39 & 10 & 5 \\
Dalinette $_{\text {Crimson }}$ & 9 & 14 & 17 & 3 \\
Belchard & 4 & 5 & 12 & 4 \\
Pink Lady & 4 & 2 & 8 & 1 \\
Garance & 1 & 1 & 1 & 1 \\
GoldRush & $7(3)$ & 9 & 10 & $4(2)$ \\
\hline Total & 1 & 0 & 2 & 0 \\
\hline
\end{tabular}




\section{Table 2 (on next page)}

Characteristics of primer pairs used in this study for multi-locus sequence typing (MSLT) identification of isolates and specific PCR. 
1 Table 2. Characteristics of primer pairs used in this study for multi-locus sequence typing (MSLT) identification of isolates and specific

2 PCR.

\begin{tabular}{|c|c|c|c|c|c|}
\hline $\begin{array}{l}\text { Locus / } \\
\text { function }\end{array}$ & Primer & Primer sequence $\left(5^{\prime}-3^{\prime}\right)$ & Reference & $\begin{array}{c}\text { Annealing } \\
\text { temperature } \\
\left({ }^{\circ} \mathrm{C}\right)\end{array}$ & $\begin{array}{c}\text { Amplicon length } \\
\text { (pb) }\end{array}$ \\
\hline \multirow[t]{2}{*}{$\begin{array}{l}\text { Alta-1 / Alternaria major allergen } \\
1\end{array}$} & Alt-for & ATGCAGTTCACCACCATCGC & $\begin{array}{l}\text { (Hong et al. } \\
\text { 2005) }\end{array}$ & 57 & 472 \\
\hline & Alt-rev & ACGAGGGTGAYGTAGGCGTC & & & \\
\hline \multirow[t]{2}{*}{ EndoPG / Endopolygalacturonase } & PG3 & TACCATGGTTCTTTCCGA & $\begin{array}{l}\text { (Andrew et al. } \\
\text { 2009) }\end{array}$ & 56 & 464 \\
\hline & PG2b & GAGAATTCRCARTCRTCYTGRTT & & & \\
\hline \multirow[t]{2}{*}{$\begin{array}{l}\text { OPA } 10-2 \text { / Anonymous } \\
\text { noncoding region }\end{array}$} & OPA10-2L & TCGCAGTAAGACACATTCTACG & $\begin{array}{c}\text { (Andrew et al. } \\
\text { 2009) }\end{array}$ & 62 & 634 \\
\hline & OPA10-2R & GATTCGCAGCAGGGAAACTA & & & \\
\hline \multirow[t]{2}{*}{$\begin{array}{l}A M T-1 \text { / Non-ribosomal peptide } \\
\text { synthethase }\end{array}$} & LinF1 & TATCGCCTGGCCACCTACGC & $\begin{array}{l}\text { Johnson et al. } \\
\qquad(2000)\end{array}$ & 65 & 496 \\
\hline & LinR & TGGCCACGACAACCCACATA & & & \\
\hline \multirow[t]{2}{*}{$A M T-2$ / Aldo-keto reductase } & AMT2-f2 & GTTGCAGAATCGCAAACTCA & $\begin{array}{l}\text { (Roberts et al. } \\
\text { 2012) }\end{array}$ & 57 & 653 \\
\hline & AMT2-r2 & GGCTCTTGGTCTCAAATCCA & & & \\
\hline \multirow[t]{2}{*}{$A M T-14$ / Unknown function } & $\begin{array}{l}\text { AMT14- } \\
\text { EMR-F }\end{array}$ & TTTCTGCAACGGCGKCGCTT & $\begin{array}{l}\text { Armitage et al. } \\
\qquad(2015)\end{array}$ & & \\
\hline & $\begin{array}{l}\text { AMT14- } \\
\text { EMR-R }\end{array}$ & TGAGGAGTYAGACCRGRCGC & & 66 & 436 \\
\hline
\end{tabular}




\section{PeerJ}




\section{Figure 1}

Bayesian phylogenetic tree of Alta-1 and EndoPg markers. The tree was constructed with sequences of 352 Alternaria isolates (261 sequences were generated in this study).

The color legend refers to the Bayesian posterior probabilities of the tree nodes. Alternaria alternata isolates are shown in blue. The Alternaria arborescens SC isolates are shown in red. Isolates from other taxonomic groups of the Alternaria section Alternaria are represented in orange (Alternaria alstroemeriae), purple (Alternaria gaisen), brown (Alternaria longipes) and grey (Alternaria gossypina). Isolates used in pathogenicity tests are highlighted in a yellow background. 


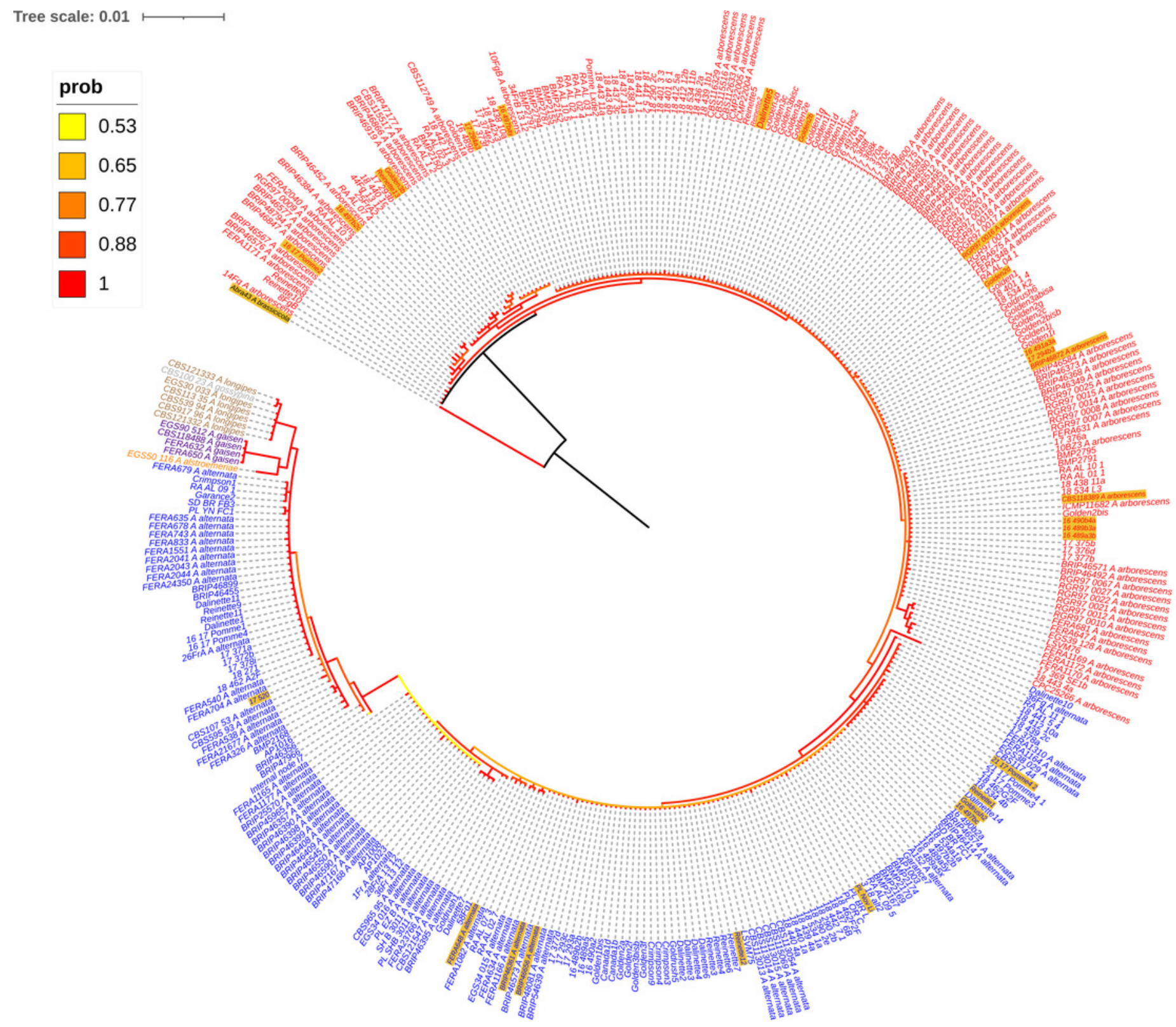




\section{Figure 2}

Mean number of leaf lesions per cultivar (Gala in red and Golden Delicious in blue) 4 days post-inoculation ( $4 \mathrm{dpi}$ ).

Results are reported for isolates of Alternaria alternata, Alternaria arborescens SC and Alternaria brassicicola, which were identified by multi-locus sequence typing (MLST). Pathogenicity tests were performed on unwounded abaxial leaf surfaces with six separate point inoculations of $10 \mu \mathrm{l}$ of Alternaria conidial suspensions (concentration of $1 \times 105$ conidia/ $\mu \mathrm{L})$. Statistical tests were only performed on Alternaria alternata and Alternaria arborescens SC. No differences were observed between isolates of Alternaria alternata and Alternaria arborescens SC or the apple cultivar (Golden Delicious and Gala). A significant effect of the experiment repetition (Type II Wald; $\chi^{2}=17.75 ; p<0.001$ ) was observed.

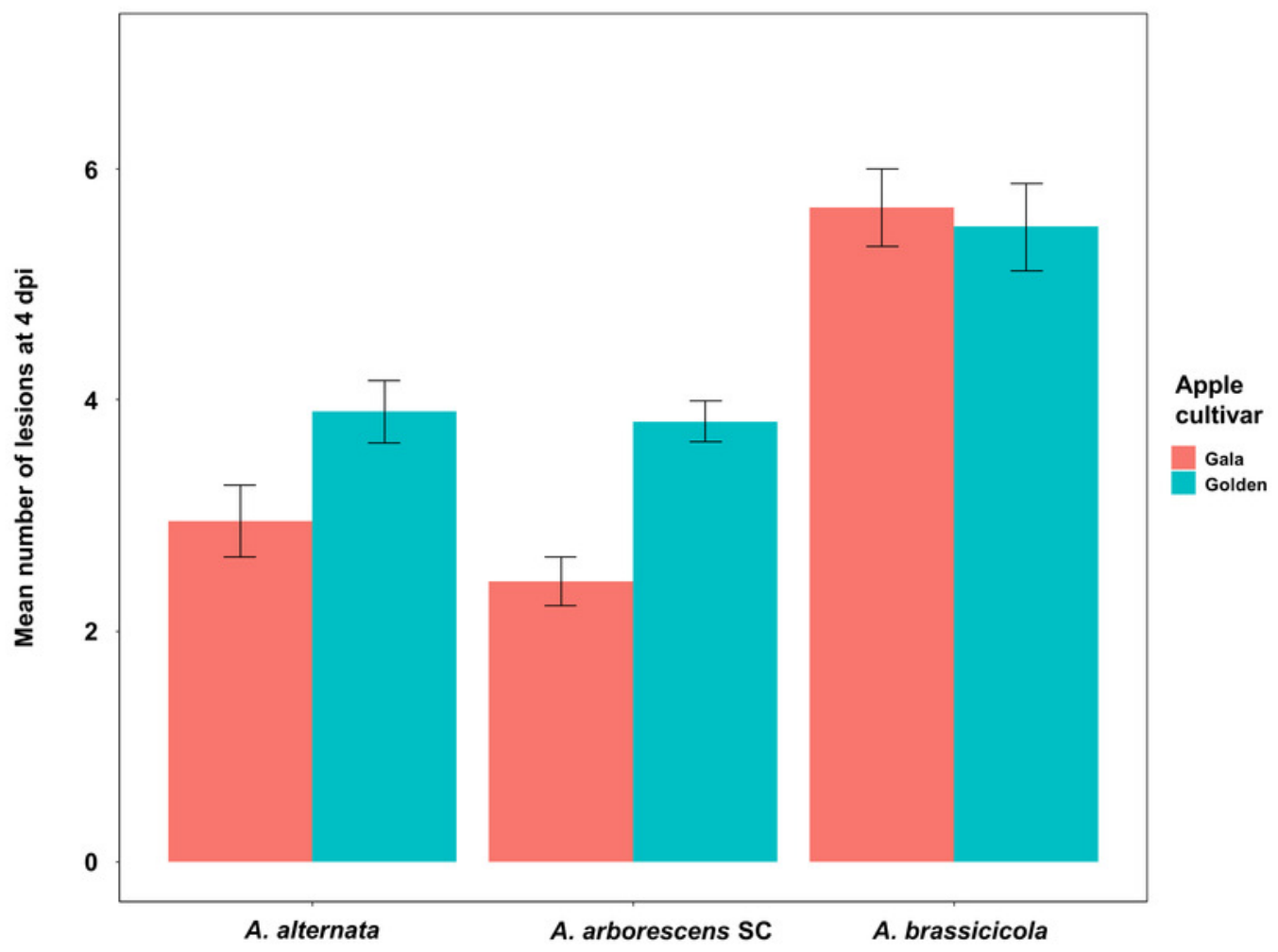




\section{Figure 3}

Mean proportion of the diseased leaf area per variety 7 and 10 days post-inoculation ( 7 , $10 \mathrm{dpi}$ ) reported for Alternaria alternata, Alternaria arborescens SC and Alternaria brassicicola.

a. Mean proportion of the diseased leaf area per cultivar (Gala in red and Golden Delicious in blue) 7 days post-inoculation ( $7 \mathrm{dpi}$ ) reported for isolates of Alternaria alternata, Alternaria arborescens SC and Alternaria brassicicola, identified by multi-locus sequence typing (MLST). Statistical tests were only performed on Alternaria alternata and Alternaria arborescens SC. The results showed that on $7 \mathrm{dpi}$, leaves of the Golden Delicious cultivar were more susceptible than leaves of the Gala cultivar (Type II Wald; $\chi^{2}=17.296 ; p<0.001$ ). No differences were observed between isolates of Alternaria alternata and Alternaria arborescens SC. A significant effect of the experiment repetition (Type II Wald; $\chi^{2}=84.013$; $p<0.001$ ) was also observed. b. Mean proportion of the diseased leaf area per cultivar (Gala in red and Golden Delicious in blue) 10 days after inoculation (10 dpi) reported for isolates of Alternaria alternata, Alternaria arborescens and Alternaria brassicicola, identified by multilocus sequence typing (MLST). Statistical tests were only performed on Alternaria alternata and Alternaria arborescens SC. The results showed that on $10 \mathrm{dpi}$, leaves of the Golden Delicious cultivar were more susceptible than leaves of the Gala cultivar (Type II Wald; $\left.\chi^{2}=11.80 ; p<0.001\right)$. No differences were observed between isolates of Alternaria alternata and Alternaria arborescens SC. A significant effect of the experiment repetition (Type II Wald; $\left.\chi^{2}=91.82 ; p<0.001\right)$ was also observed. 


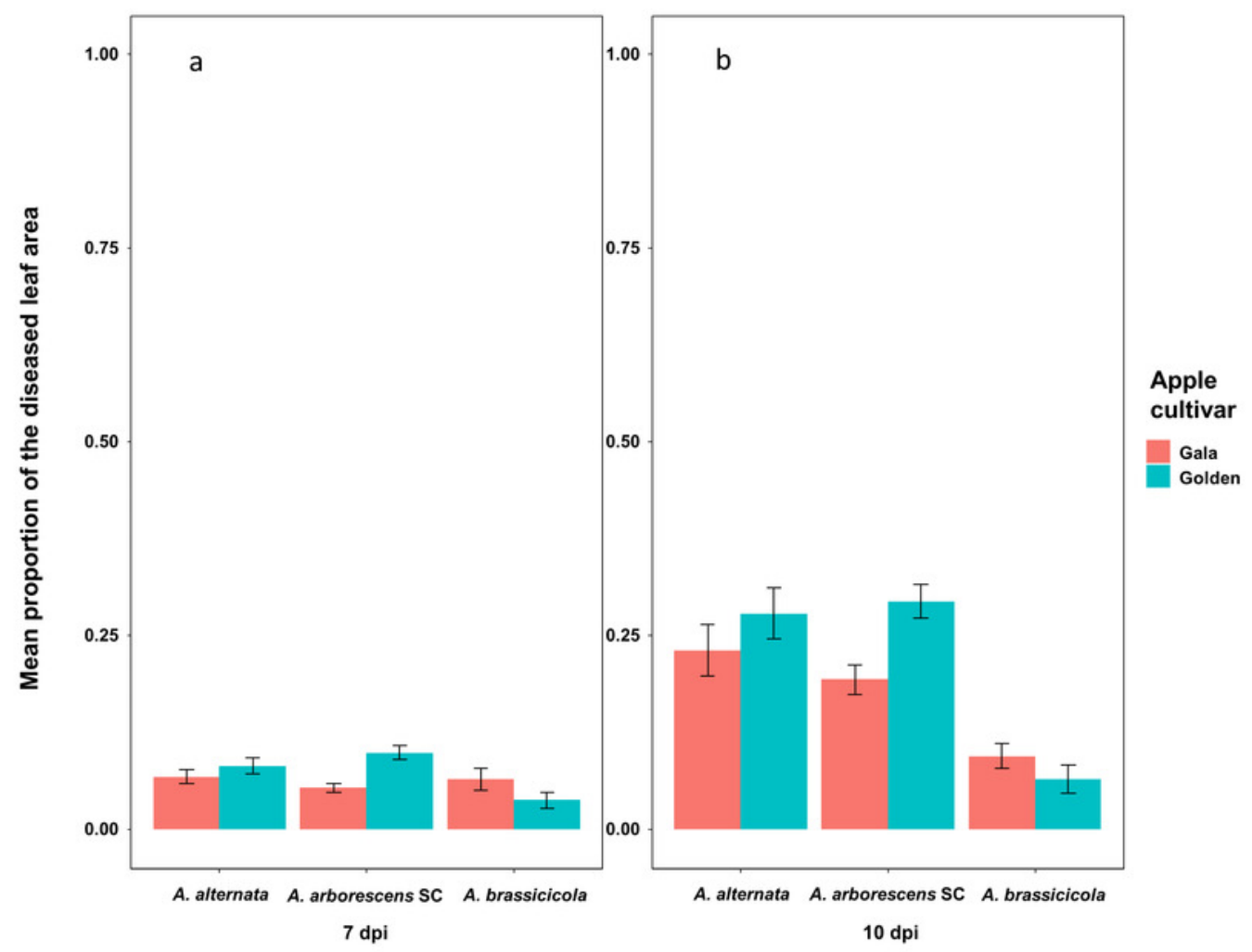

